\title{
LA CONTINUIDAD EN LAS OBRAS DE NIETZSCHE A LA LUZ DE SU VISIÓN DE SÍ MISMO ${ }^{1}$

\author{
Continuity in Nietzsche's works in light of his self-view
}

\author{
Marina García-Granero \\ Universitat de València
}

\begin{abstract}
RESUMEN: El artículo tiene como objetivo analizar críticamente la convencional división tripartita de la filosofía de Nietzsche que en su forma hiperbólica se expresa en forma de personificación: el Nietzsche joven de la fase wagneriana, el Nietzsche «positivista»o «ilustrado» y el Nietzsche maduro. Se propone contribuir al alegato en favor de la continuidad, prestando especial atención a una variedad de textos en los que Nietzsche contempla la continuidad de su desarrollo intelectual como una unidad coherente. Esta significativa retrospección no indica ruptura o rechazo, sino reconocimiento. Dedicaremos la última sección al peculiar libro que es Ecce Homo, entendido como estrategia de auto-presentación.
\end{abstract}

Palabras clave: continuidad - periodización - fase wagneriana - Ecce Homo autopresentación

ABSTRACT: The article aims to critically analyze the conventional tripartite division within Nietzsche's philosophy that is expressed hyperbolically in the form of personification: the young Nietzsche of the Wagnerian phase, the "positivist" or "enlightened" Nietzsche, and the mature Nietzsche. I aim to contribute to the plea for continuity, paying special attention to a variety of texts in which he acknowledges the continuity of his intellectual development as a coherent unit. This significant retrospection does not indicate rupture or rejection, but recognition. The last section is dedicated to the peculiar book that is Ecce Homo, understood as a self-presentation strategy.

Keywords: continuity - periodization - Wagnerian phase - Ecce Homo - self-presentation

1 Esta publicación ha recibido el apoyo del proyecto de Investigación Científica y Dsarrollo PID2019-109078RB-C22 financiado por el Ministerio de Ciencia e Innovación. La autora agradece asimismo el soporte de una ayuda a contratos FPU (referencia FPU15/04085) del Ministerio de Universidades. 


\section{INTRODUCCIÓN}

Uno de los lugares comunes más extendidos de la comprensión mayoritaria de la filosofía de Nietzsche es su periodización. Como es sabido, esta división funcionaría del siguiente modo: en primer lugar, de los años de formación hasta las Consideraciones Intempestivas, en segundo lugar, el período intermedio «positivista» o ilustrado ${ }^{2}$ — según las distintas interpretaciones-, que se correspondería con las obras escritas en estilo aforístico, desde Humano, demasiado humano hasta La gaya ciencia, y el último período iría desde Asi habló Zaratustra hasta la pérdida de la conciencia al comienzo de 1889.

Esta tripartición, cuando excede meros propósitos pragmáticos, posee, a mi juicio, un efecto indeseado de crear en ocasiones - no sólo, pero al menosdos tipos distintos de intérpretes: los que privilegian la primera fase, a menudo más interesados en una lectura estética de su filosofía, y los especialistas del período de madurez, a menudo más interesados por la vertiente o la crítica moral, política y social, mientras que el llamado período intermedio suele quedar en un segundo plano. Además, el público filosófico general, que asimila esta tripartición, suele llegar a la apreciación de que Nietzsche o bien posee interés únicamente como esteta o bien es un irracionalista que se contradice continuamente. En realidad, la filosofía de Nietzsche no es contradictoria, simplemente progresa ${ }^{3}$. A mi modo de ver, la apreciación de la contradicción desde un punto de vista metodológico surge cuando no se lee a Nietzsche con el rigor necesario, atendiendo a los diversos escritos - obras publicadas, manuscritos, escritos o fragmentos póstumos, correspondencia, siempre manteniendo a cada uno en su estatuto ${ }^{4}$. Como expresa Nietzsche en el prefacio de 1886 al segundo volumen de Humano, demasiado humano, sus escritos son la historia de sus superaciones ${ }^{5}$, en las que añade, modifica o rechaza las posturas y tesis defendidas anteriormente, y en este movimiento radica la riqueza del pensamiento. Así se puede interpretar también la crítica o discernimiento de Nietzsche de sus anteriores obras, como veremos en los

2 Eugen Fink, Nietzsches Philosophie, Stuttgart: Kohlhammer, 1968, p. 42. Edición española: La filosofia de Nietzsche, trad. de Alberto Ciria, Barcelona: Herder, 2019. Para una interpretación crítica con la consideración de ilustrado y con la valoración misma del «período intermedio», cf. Diego Sánchez Meca, El itinerario intelectual de Nietzsche, Madrid: Tecnos, 2018, pp. 162-168.

3 FP II 851, 12[128]: «Hoy estás en contra de lo que ayer aprendiste — Mas por eso ayer no es hoy, dijo Zaratustra».

4 Cf. Manuel Barrios Casares, «Nietzsche: una inflexión decisiva en el país de la discontinuidad»: Estudios Nietzsche, 18 (2018), 13-27.

5 HDH II «Prólogo» §1, OC III 276: «Sólo se debe hablar cuando ya no se puede callar; y hablar sólo de aquello que se ha superado, — cualquier otra cosa es charlatanería, "literatura", falta de disciplina. Mis escritos sólo hablan de mis superaciones: "yo" estoy dentro de ellos, con todo aquello que fue enemigo mío, ego ipsissimus, e incluso, si se me permite una expresión más orgullosa, ego ipsissimum. Se adivina: tengo ya muchas cosas — debajo de mí...». 
distintos ejemplos, más allá del caso paradigmático del «Ensayo de autocrítica» (1886) incorporado a la tercera edición de El nacimiento de la tragedia ${ }^{6}$.

Frente a la tendencia de la tripartición, que parece crear una hipóstasis de tres «Nietzsche» distintos, cuyas filosofías serían radicalmente contrarias, coincido con los especialistas que, como Sánchez Meca, han argumentado que en el pensamiento de Nietzsche hay un itinerario coherente, «una coherencia que justifica los cambios» ${ }^{7}$, una dirección dentro de un mismo proceso de superación, un «destino», como el propio Nietzsche expresa en Ecce Homo. Sus escritos constituyen una unidad en devenir, por tanto, la continuidad posea mayores virtualidades hermenéuticas que la discontinuidad ${ }^{8}$. La edición de las Obras Completas en Tecnos, dirigida por Sánchez Meca, no reconoce intermezzo alguno entre juventud y madurez, pues se considera con razón que dicha madurez ya se alcanza en Humano, demasiado humano ${ }^{9}$. De forma similar, a mi juicio, podemos designar textos que comparten problemáticas, enfoques o estilo, como en el caso de los aforísticos - los habitualmente considerados como «intermedios» - , pero siempre siendo conscientes de que esta distinción es una ficción, porque no hay Nietzsches distintos, ni filosofías radicalmente diferentes, opuestas o contradictorias entre sí, sino una vida y una filosofía en desarrollo.

En el seno de nuestro alegato a favor de la continuidad, ya impulsado por reputados especialistas, otorgaremos especial importancia a lo que Nietzsche expresa sobre esta cuestión, es decir, su visión de sí mismo y su percepción de su trayectoria, sus reflexiones cuando echa la vista atrás y medita sobre sus escritos y su biografía. En este análisis la correspondencia es indispensable para estudiar su narración de sí, como una herramienta hermenéutica inestimable que permite comprender mejor lo que hay detrás de sus escritos, su proceso de escritura y cómo Nietzsche «se autointerpreta en su propia filosofía» ${ }^{10}$. Es un soporte propicio para la exploración sobre la propia identidad, dentro de la intimidad de las conversaciones con sus amistades y seres queridos,

6 NT «Ensayo de autocrítica» §3, OC I 331. Cf. Miguel Ángel de Barrenechea, «Ensayo de autocrítica: una perspectiva genealógica sobre la primera obra de Nietzsche»: Estudios Nietzsche 17 (2017), 31-42.

7 Diego Sánchez Meca, op. cit., p. 153.

8 También en su pensamiento político, cf. Manuel Knoll, «The 'Übermensch' as a Social and Political Task: A Study in the Continuity of Nietzsche's Political Thought», en Manuel Knoll y Barry Stocker (eds.), Nietzsche as Political Thinker, Berlin/Boston: de Gruyter, 2014, pp. 239-266.

9 José Emilio Esteban Enguita, «Del arte de guiar: apuntes sobre las introducciones de las Obras Completas en español de F. Nietzsche»: Estudios Nietzsche 18 (2018), 29-39.

10 Luis Enrique de Santiago Guervós, «La Correspondencia de Nietzsche: la otra cara del 'Ecce Homo'»: Estudios Nietzsche 18 (2018), 95-113, p. 94. Maria Cristina Fornari, «'And so I Will Tell Myself the Story of my Life'. Nietzsche in His Last Letters (1885-1889)», en João Constâncio y Maria João Mayer Branco (eds.), As the Spider Spins. Essays on Nietzsche's Critique and Use of Language, Berlin/Boston: de Gruyter, 2012. 
además de las conversaciones con los editores a través de las cuales gesta sus obras. Se plasma la reflexión de Nietzsche sobre la vertebración de sus obras y su desarrollo intelectual, en la diversidad de sus escritos con el objetivo de mostrar cómo en ellos la única fase reconocida explícitamente es la wagneriana, y cómo, por lo demás, prima con claridad una perspectiva que reconoce la continuidad. Dedicaremos la última sección al peculiar libro que es Ecce Homo, entendido como estrategia de auto-presentación.

\section{SOBRE LA FASE WAGNERIANA Y LOS «CENTAUROS»}

La clásica distinción tripartita fue interpretada, especialmente al comienzo del siglo pasado, en un sentido hegeliano como sucesión de tres períodos de tesis, antítesis y síntesis ${ }^{11}$. Dicha interpretación se basaría en un hipotético retorno de Nietzsche a posiciones metafísicas en su período maduro, bajo el supuesto de una contigüidad entre El nacimiento de la tragedia y Así habló Zaratustra tomando como ejemplo la doctrina del eterno retorno, o una presumida analogía entre la voluntad de vivir de Schopenhauer y la voluntad de poder nietzscheana ${ }^{12}$. Por otro lado, son varios los especialistas que han criticado que esta periodización tripartita contribuye, además, a minimizar la importancia del período intermedio ${ }^{13}$. En realidad, como ha mostrado con explicitud Sánchez Meca, el itinerario filosófico de Nietzsche se articula en una continuidad, una progresión, en la que todos los pasos recorridos han resultado ser fundamentales ${ }^{14}$. Me propongo contribuir a las argumentaciones ya realizadas a favor de la continuidad, con el análisis de una selección de textos en los que Nietzsche expresa su visión de sí mismo.

Como ya han destacado con acierto especialistas como Montinari o D'Iorio, la única fase que Nietzsche reconoce es la wagneriana. «Fase»

11 Heinrich Römer, Nietzsche, Leipzig: Klinkhardt \& Biermann, 1921, I, p. 105 ss., y August Vetter, Nietzsche, der Verkünder neuer Ziele, Munich: Reinhardt, 1926, p. 158, citados por Richard Roos, «Les derniers écrits de Nietzsche et leur publication»: Revue Philosophique de la France et de l'Étranger, 146 (1956), 262-287.

12 Cf. la crítica de Mazzino Montinari, «Nietzsche contra Wagner: été 1878», en Marc Crépon (ed.), Nietzsche, col. Cahiers de l'Herne, Paris, L'Herne, 2000, pp. 237-244, p. 237. Esta interpretación en clave hegeliana disminuye la importancia de los llamados escritos intermedios. Son varios los textos que contradicen esta hipótesis, por ejemplo, Nietzsche sostiene en una carta a Malwida von Meysenbug haber escrito «el comentario antes del texto», en referencia a su Zaratustra: Carta ${ }^{\circ} 404$ de 1883, CO IV p. 348. Además, el eterno retorno aparece ampliamente teorizado en el cuaderno $\mathrm{M}$ III 1 de 1881, un par de años antes del primer libro de Zaratustra.

13 Cf. Montinari en el artículo ya citado, y Paolo D'Iorio, «Système, phases diachroniques, strates synchoniques, chemins thématiques», en Paolo D’Iorio y Olivier Ponton (eds.), Nietzsche. Philosophie de l'esprit libre. Études sur la genèse de Choses Humaines, trop Humaines, Paris, Éditions Rue d'Ulm - Presses de l'École Normale Supérieure, 2004, pp. 22-36, p. 26.

14 Diego Sánchez Meca, op. cit., la «Nota final», p. 291, además de distintas secciones del libro expresamente dedicadas al tratamiento de la continuidad. 
(Phase) es, de hecho, un término que emplea en dos ocasiones para referirse a los años del período wagneriano. Una de las ocasiones en que se refiere a esta «fase» es una carta a Mathilde Maier de 1878:

$[+++]$ de la grandeza de Wagner pocos pueden estar tan persuadidos como yo: porque pocos saben tanto de ella. Sin embargo he pasado de ser un partidario sin reservas, a ser uno con reservas: como lo somos con respecto a todos los grandes del pasado; como lo soy con respecto a la fase de los últimos diez años de mi vida - la apruebo en su conjunto, pero ahora conozco un punto de vista superior. Por lo que respecta a Wagner, había visto lo superior, su ideal — con ello llegué a $\mathrm{B}<$ ayreuth $>$ - por ello mi desilusión ${ }^{15}$.

Este fragmento expresa una cierta aquiescencia con respecto a la fase wagneriana y a la grandeza de Wagner, que no puede asimilarse a un rechazo frontal, sino más bien a un desencantamiento. Formula el reconocimiento de que la fase fue necesaria para acceder a una posición superior. Unos años más tarde, en una anotación póstuma de 1883, Nietzsche habla del «jesuitismo» de su «primer período»: «la persistencia consciente en la ilusión y la incorporación forzosa de la misma como base de la cultura. O bien: budismo y anhelo de la nada (la contradicción schopenhaueriana entre teoría y práctica, insostenible). Wagner no ha superado el primer peligro» ${ }^{16}$. Mediante «jesuitismo», Nietzsche expresa la estrategia de «afirmar aquello en lo que no se cree para provocar el advenimiento de una situación que se sostendrá en una mentira o una ilusión ${ }^{17}$, es decir, la cultura basada en la ilusión y la bella mentira del arte de Wagner. Por tanto, en su caso específico se referiría a su adhesión a la causa wagneriana y su proyecto de transformación de la cultura a través de la metafísica de artista, de la cual se emanciparía después con su propuesta alternativa.

Pero para entender esta fase, por supuesto, hay que remontarse a su comienzo. Al final de sus estudios universitarios, Nietzsche sintió el traslado gradual de su interés por la filología hacia la filosofía materialista. Fruto de su deseo de ampliar sus conocimientos filosóficos, se embarca en el verano de 1866 en la lectura de un libro clave en la posterior orientación de su filosofía: la Historia del materialismo y crítica de su significado para el presente del neokantiano Friedrich Albert Lange ${ }^{18}$. Esta obra le causa una gran impresión y le impulsó a estudiar a Kant a través de la Historia de la filosofía moderna de

15 Carta $^{\circ} 741$ de 1878,741, CO III 298-299. Cf. FP II 386, 27[65]: «En el bosque de Bohemia me elevé a una fase superior».

16 FP III 350, 16[23].

17 FP III 350, cf. nota a pie de página 29 de los traductores Diego Sánchez Meca y Jesús Conill.

18 Friedrich Albert Lange, Geschichte des Materialismus und Kritik seiner Bedeutung in der Gegenwart, Baedeker: Iserlohn, 1866. 
Kuno Fischer. Esta fecha está demostrada por la correspondencia, por ejemplo en agosto de 1866 escribe una extensa carta a Carl von Gersdorff en la que le traslada las conclusiones de su lectura de Lange ${ }^{19}$. Nietzsche aprecia en Lange un doble carácter como «kantiano ilustrado» y también «investigador de la naturaleza» ${ }^{20}$. En este punto la orientación antimetafísica de Lange no es considerada aún motivo suficiente para abandonar a Schopenhauer, pues «incluso en este punto de vista crítico de lo más estricto siempre nos queda nuestro Schopenhauer» ${ }^{21}$. Se aprecian reticencias a romper con su maestro, cuya obra es comparada a través de un símil artístico con Beethoven o Rafael, para después concluir: «yo no conozco a ningún filósofo que edifique más que nuestro Schopenhauer» ${ }^{22}$. Pero será sólo cuestión de tiempo, puesto que la obra de Lange despertó su interés en Demócrito.

Entre otoño de 1867 y la primavera de 1868, mientras cumplía su servicio militar, redactó una serie de estudios sobre la teleología ${ }^{23}$, el concepto de orgánico de Kant a nuestros días, y sobre el materialismo de los antiguos y los modernos ${ }^{24}$, en especial sobre Demócrito ${ }^{25}$, a quien dedica numerosas notas filosóficas e histórico-filológicas, paralelas a las dedicadas a la sucesiva crítica de Schopenhauer. Demócrito se convierte, a juicio de Montinari, en una especie de figura anti-Schopenhaueriana, por su orientación teórica completamente opuesta, agnóstica y antimetafísica ${ }^{26}$.

Pero entonces Nietzsche gana la cátedra de Basilea a los 24 años, y sus idas y venidas entre Basilea y la residencia de los Wagner en Tribschen lo empujan al fervor de la causa wagneriana. Publica El nacimiento de la tragedia en 1872, que le proporciona celebridad dentro de los círculos wagnerianos y una relación estrecha con Wagner. En esta obra ampara una metafísica del arte y del artista de acuerdo con la cual la existencia sólo es digna de ser vivida desde una perspectiva estética. En dicha metafísica del artista, la sociedad está consagrada al genio, que funda y salvaguarda la comunidad, gracias a la función consoladora y redentora del mito. Pero incluso en este período las reflexiones sobre los filósofos antiguos ocupan su atención, como muestran las lecciones sobre Los filósofos preplatónicos, aunque permanecían en un segundo plano con respecto a su actividad pública. La fase wagneriana se

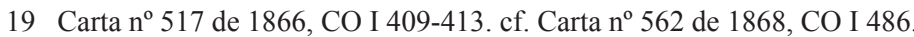

20 Ibid., p. 412.

21 Ibid., p. 413.

22 Ibid.

23 Cuaderno P I 8 «Sobre la teleología», OC I 304-320.

24 Paolo D'Iorio, Le voyage de Nietzsche à Sorrente, Paris: CNRS Éditions, 2015, p. 86. Edición española: Paolo D'Iorio, El viaje de Nietzsche a Sorrento, trad. de Luis Enrique de Santiago Guervós, Barcelona: Gedisa, 2016.

25 «Apuntes para un ensayo sobre Demócrito», en OC I 241-292.

26 Mazzino Montinari, Che cosa ha detto Nietzsche, Milano, Adelphi, 1999, p. 64. 
caracteriza por una profunda escisión entre lo que el joven profesor publica en sus obras, y lo que escribe en sus cuadernos privados o confía a sus estudiantes ${ }^{27}$, es decir, aquello en que realmente pensaba o aquello que le interesaba. En marzo de 1873 escribe otra carta a Gersdorff en la que habla de un escrito en curso sobre los filósofos preplatónicos, y que va asumiendo «el aspecto de un apéndice a la "tragedia". El título será quizás: El filósofo como médico de la cultura. Con este trabajo querría darle una verdadera sorpresa a Wagner en su cumpleaños» ${ }^{28}$. La tarde del 7 de abril, Nietzsche comienza a leerle dicho escrito, acompañados por Cosima Wagner y Erwin Rohde ${ }^{29}$. La respuesta de Wagner fue fría y su influjo era tan fuerte que su atención se desvió entonces al proyecto de las Consideraciones inactuales, empezando por la dedicada a David Strauss ${ }^{30}$, un proyecto mucho más afín a la causa wagneriana.

En otoño de 1876, Nietzsche solicita a la Universidad de Basilea un año sabático por sus problemas de salud, y se embarca en su primer viaje al sur de Europa, en concreto a Sorrento, en Italia. Como ha estudiado Paolo D'Iorio, este viaje posee una importancia excepcional tanto en el sentido biográfico como en el filosófico, en concreto, en lo que respecta a la orientación de su filosofía y el giro antimetafísico. Es en Sorrento donde Nietzsche decide convertirse en filósofo ${ }^{31}$, escribir y publicar libros genuinamente filosóficos, abandonar su cátedra de Filología en Basilea y cambiar de vida por completo. También ahí escribirá la mayoría de los aforismos de Humano, demasiado humano, libro dedicado a Voltaire que anuncia el comienzo de su filosofía emancipada. Desde el punto de vista estilístico, se aprecia el tránsito en los cuadernos de Sorrento hacia el aforismo, y que conducirá a la publicación de las obras en estilo aforístico - Humano, demasiado humano, Aurora, La gaya ciencia - , y desde el punto de vista filosófico, recupera elementos de su formación filosófica y filológica, que en realidad llevaba explorando de forma privada o en sus lecciones de Basilea. Desaparece, así, la escisión entre lo que publica y lo que escribe en sus cuadernos. En 1876 Nietzsche renuncia a la causa wagneriana y pone fin a esta fase de diez años. En los cuadernos

27 Paolo D'Iorio, Le voyage de Nietzsche à Sorrente, cit., p. 14.

28 Carta no $^{\circ} 298$ de 1873, CO II p. 392.

29 Paolo D'Iorio, «La naissance de la philosophie enfantée par l'esprit scientifique», en Friedrich Nietzsche, Les philosophes préplatoniciens, Paris: 1'Éclat, 1994, pp. 11-49, p. 39.

30 Carta $^{\circ} 304$ de 1873, CO II p. 402.

31 Paolo D'Iorio, Le voyage de Nietzsche à Sorrente, cit., p. 17. 
redactados en Sorrento podemos encontrar varios apuntes en los que plasma esta evolución y declara su nueva autonomía:

A los lectores de mis escritos precedentes quiero manifestar de forma expresa que he abandonado los puntos de vista metafísico-artísticos que en esencia dominaban en ellos: son agradables, pero insostenibles. Quien se permite hablar en público en fecha temprana, por lo habitual se ve obligado al poco tiempo a contradecirse públicamente ${ }^{32}$.

Un año antes ya había anticipado en sus cuadernos la ruptura con los círculos wagnerianos, una ruptura que no sólo fue llevada a cabo en lo intelectual sino también en lo personal, con el conjunto de amistades creadas en ellos ${ }^{33}$.

El capítulo de Ecce Homo dedicado a Humano, demasiado humano es especialmente explícito al respecto de este retorno a la filosofía. Como es sabido, ahí Nietzsche escribe que Humano, demasiado humano «es el monumento a una crisis», a partir de él se libera de «lo no perteneciente a [su] naturaleza» ${ }^{34}$. Es el testimonio de «un espíritu que se ha hecho libre, que ha vuelto a tomar posesión de sí mismo» ${ }^{35}$. Puntualiza además que en las semanas del festival de Bayreuth la ruptura con Wagner fue sólo un elemento de una multitud de percepciones e intuiciones sobre su vida:

De repente me quedó claro, con terrible nitidez, cuánto tiempo se había desperdiciado ya, - de qué forma tan inútil, tan arbitraria, toda mi existencia de filólogo me había desviado de mi tarea. Me avergoncé de esa falsa modestia... Había dejado detrás de mí diez años en los que la nutrición de mi espíritu había estado prácticamente suspendida por completo, en los que no había aprendido nada de provecho, en los que había olvidado de manera absurda muchas cosas a cambio de unos chismes de polvorienta erudición ${ }^{36}$.

Los estudios nietzscheanos muestran con claridad que después de la fase wagneriana, tras el abandono del idealismo y la fe metafísica, la reflexión incorpora las disciplinas históricas, científicas y psicológicas, si bien se sabe que por los póstumos, en especial aquellos que son apuntes de lectura, que ya llevaba muchos años cultivando esta amplia diversidad de disciplinas, más

32 FP II 361, 23[159].

33 FP II 101, 5[190]: «He de manifestar en breve opiniones que se consideran ignominiosas para quien las sostiene; hasta los amigos y conocidos se mostrarán entonces esquivos y medrosos. También he de atravesar este fuego. Tras ello, cada vez me perteneceré más a mí mismo», posteriormente publicado con ligeras modificaciones y en voz impersonal, en HDH §619, OC III 263-264.

34 EH «Humano, demasiado humano» §1, OC IV 825.

35 Ibid.

36 EH «Humano, demasiado humano» $\S 3$, OC IV 827. 
allá de la filología. El elemento novedoso reside en que estas disciplinas y reflexiones salen a la luz, ocupando su debido espacio, tras la fase wagneriana.

Desde Sorrento, Nietzsche confiesa a Cosima Wagner en una carta del 19 de diciembre de 1876, con motivo de su cumpleaños, que: «ya cuando escribía sobre $\mathrm{Sch}<$ openhauer $>$, me di cuenta de que había superado toda la parte dogmática; para mí el hombre lo era todo» ${ }^{37}$. También reflexiona sobre los últimos ocho años y expresa que es consciente de haber «llegado mucho más allá de las esperanzas y deseos generales de la juventud», posible referencia al logro de la cátedra con veinticuatro años, pero «por el contrario, de todo aquello que me había propuesto expresamente hacer, sólo he sido capaz de realizar un tercio como promedio» ${ }^{38}$, es decir, de los proyectos que abandonó ocho años atrás, en 1868. Sabemos por los escritos póstumos que ya en la época del servicio militar, en 1867, mucho antes de Schopenhauer como educador (1874), Nietzsche redacta en sus cuadernos privados una refutación detallada de la filosofía de Schopenhauer ${ }^{39}$, notas que ya anunciaban Humano, demasiado humano. La crítica de Wagner se intuía ya en la cuarta Consideración intempestiva, que en uno de los prólogos de 1886 es descrita al mismo tiempo como un «gesto de gratitud hacia un trozo de mi pasado», «una separación, una despedida» ${ }^{40}$. Como mostró Montinari, «Richard Wagner en Bayreuth», representa un «retrato», un «mosaico extremadamente hábil» de citas wagnerianas ${ }^{41}$, con las que Nietzsche rescataba el sentido originario del proyecto wagneriano, el Wagner ateo, revolucionario y discípulo de Feuerbach, para contrastarlo con la «puesta en escena» actual del drama sagrado, el Wagner impostor, de la redención, germánico y romántico. Antes del festival, el texto parecía un manifiesto wagneriano, pero después de él era simplemente una evidencia de la discordancia y el contraste radical entre el proyecto expresado en los escritos feuerbachianos y el espectáculo de Bayreuth. Más tarde, en Ecce Homo encontramos otra significativa referencia a este texto, en el que no se aprecia rechazo, sino que reconoce en el retrato del artista ditirámbico «la imagen preexistente del poeta del Zaratustra». Sostiene que «se puede poner "Zaratustra" sin recelo alguno ahí donde en el texto pone la palabra "Wagner" $\rangle^{42}$, recalcando así cómo la crítica se sitúa en la discrepancia entre el ideal y la forma que malogró el proyecto wagneriano.

37 Carta $^{\circ} 581$ de 1976, CO III 191-193. Cf. FP II 388, 27[80].

38 Carta n $^{\circ} 581$ de 1876, CO III p. 192. En FP II 394, 28[33]: «En Sorrento levanté la capa de moho de los nueve años. Soñar con muertos».

39 Cuaderno P 16 «Sobre Schopenhauer» (1867), en OC I 293-300.

40 HDH II «Prólogo» §1, OC III 276.

41 Mazzino Montinari, «Nietzsche und Wagner vor hundert Jahren», en Nietzsche lesen, Berlin-New York: de Gruyter, pp. 38-55, p. 45.

$42 \mathrm{EH}$ «El nacimiento de la tragedia» §4, OC III 820. 
Sobre la base de esta vuelta a sí mismo y la recuperación de la filosofía, D'Iorio sugiere que en realidad se podría «poner entre paréntesis la fase wagneriana e instaurar una fuerte continuidad entre las primera reflexiones de los escritos de juventud y la filosofía del espíritu libre», y que «la filosofía de Nietzsche no comienza con la metafísica del arte de El nacimiento de la tragedia, bajo la égida de Schopenhauer y al lado de Wagner, sino con la valorización de Demócrito, el borrador de ensayo contra la teleología y una crítica despiadada de la metafísica de Schopenhauer ${ }^{43}$. Mediante esta «señalización» de la fase wagneriana no se está sugiriendo en absoluto que se deban desechar e ignorar completamente los trabajos de dicho período. A él pertenecen, por ejemplo, las lecciones sobre Los filósofos preplatónicos, aquellos filósofos que descubrieron «bellas posibilidades de vida», y de los que admira su figura y también la ausencia de carácter clerical ${ }^{44}$. En las notas póstumas de ese período encontramos de hecho una idea que devendrá un tema fundamental de la filosofía nietzscheana: la reflexión sobre «la producción (Erzeugung) y el cultivo (Bildung) de un ser humano», no abandonarse a nada similar a cualquier «providencia», sino de modo consciente fomentar su nacimiento y su florecimiento ${ }^{45}$, lo que más tarde se formulará en términos de filosofía experimental, de modos de vida más afirmativos — contra el último hombre - y la búsqueda de condiciones favorables a largo plazo para los individuos superiores o el advenimiento del superhombre, según las distintas teorizaciones.

Ya en 1875 Nietzsche escribe con tono fantasioso que «una coalición de un gran centro de hombres para la producción de mejores hombres es la tarea del futuro» ${ }^{46}$, la misma tarea que en sus últimos años otorgará a los «filósofos del futuro», empleando con explicitud los términos de la «cría». Así, en la fase wagneriana encontramos los "centauros» ${ }^{47}$, la expresión de una misma sensibilidad y reflexiones que se desarrollarán y madurarán más adelante, una vez emancipado por completo de sus maestros. Él mismo reconoce en

43 Paolo D'Iorio, op. cit., p. 27.

44 FP II 118, 6[48]. Paolo D’Iorio, «La naissance de la philosophie enfantée par l'esprit scientifique», cit.

45 FP II 61, 3[75]. Cf. FP II 113, 6[31]: «La mayor pérdida que puede sufrir la humanidad es el no llegar a realizar los tipos supremos de vida», nótese este uso temprano de «tipo».

46 FP II 61, 3[75].

47 BVN-1870,58, CO II 123: «Voy a volverme la esperanza personificada: también Richard Wagner me ha dado a conocer de la manera más conmovedora cuál es el destino que ve asignado para mí. (...) Por el contrario quiero, cuando llegue el momento, expresarme de la manera más seria y sincera posible. Ahora, dentro de mí, ciencia, arte y filosofía crecen juntos de tal forma que alguna vez, ciertamente, pariré centauros». Cf. Tilman Borsche, Federico Gerratana y Aldo Venturelli (eds.), Centauren-Geburten. Wissenschaft, Kunst und Philosophie beim jungen Nietzsche, Berlin-New York: de Gruyter, 1994. 
una anotación póstuma: «Me he ido dejando por aquí y por allá puertas de salida en las Consideraciones Intempestivas» ${ }^{48}$, que podemos interpretar como pista o alusión a ciertos pensamientos que ya se colocaban fuera de la causa wagneriana, como germen futuro de la verdadera filosofía nietzscheana, emancipada de Wagner. En otra carta a Mathilde Maier de julio de 1878 expresa cómo esta «crisis y transformación» ha supuesto su giro definitivo hacia la filosofía:

No hay remedio: estoy abocado a poner en aprietos a mis amigos — incluso cuando explico finalmente cómo he salido yo mismo de las dificultades (...) estoy decidido más que nunca en favor de todo lo bueno y capaz, cien veces más cerca de los griegos que antes: cómo ahora yo mismo vivo, hasta en el más mínimo detalle, aspirando a la sabiduría, mientras que antes sólo honraba e idolatraba a los sabios - en suma, si usted pudiera sentir como yo esa crisis y transformación, ¡oh, entonces usted desearía vivir algo similar! (...)

Ahora me sacudo todo lo que no me pertenece, personas, tanto amigos como enemigos, costumbres, comodidades, libros; viviré en soledad durante años hasta que me sea lícito (y probablemente necesario) volver a relacionarme, ya maduro y preparado, como filósofo de la vida. ${ }^{49}$

Al tenor de esta carta, los fragmentos del grupo 30 de 1878 y otros documentos que atestiguan una importante introspección y reflexión autobiográfica $^{50}$, Montinari argumentó que el verano de 1878 constituye el momento de máxima significatividad en lo que respecta a la ruptura con Wagner, inmediatamente después de la publicación de Humano, demasiado humano $^{51}$. Es también en ese verano cuando Wagner hace patente la ruptura también por su parte mediante la publicación de su escrito «Público y popularidad», publicado en los Bayreuther Blätter, revista del movimiento wagneriano. Humano, demasiado humano expresaba no sólo la emancipación de Nietzsche de los círculos wagnerianos, sino que constituía en sí misma una invitación a sus amistades a abandonarlos, como Erwin Rohde, Franz Overbeck y Malwida von Myesenbug y el propio Wagner, pero la propuesta no fue aceptada ni por Wagner ni por los demás, que simplemente achacaron

48 FP II 248, 17[36].

49 Carta no $^{\circ} 734$ de 1878, CO III p. 293.

50 Cf. FP II 414, 30[72] donde habla de sus lectores y del impacto que tendrá en ellos su cambio de gusto y estilo de escritura. Sobre las fuentes y estímulos de este impulso autobiográfico en Nietzsche desde su infancia, cf. Maria Cristina Fornari, «Talis hominibus fuit oratis qualis vita. Nietzsche y la narración de sí»: Quaderns de filosofia 2/1 (2015), 25-38.

51 Mazzino Montinari, «Nietzsche contra Wagner: été 1878», cit., pp. 238-239. 
el distanciamiento de Nietzsche al influjo de Paul Rée, «el filósofo positivista que para los wagnerianos tenía el fallo de ser judío» ${ }^{52}$.

Como puntualiza Montinari, esta fase no representa «la primera fase de una serie, sino la fase wagneriana, schopenhaueriana, que luego deja tras de sí (no hay otras fases)» ${ }^{53}$. Tras la fase wagneriana comienza la pasión del conocimiento $^{54}$ que caracteriza el conjunto de toda su filosofía y que representa un importante elemento del sentido de la misma: el abandono de todos los mitos y las fuentes de saber privilegiado — el genio, el santo, el artista-, y que se expresa en los escritos de madurez en la destrucción de los ídolos. Probablemente esta emancipación tras la fase wagneriana marcara la posterior concepción de Nietzsche respecto a la necesaria traición del discípulo con respecto a su maestro ${ }^{55}$. La superación de la «superstición del genio» ${ }^{56}$ se presenta, pues, como una autocrítica: no tanto como una polémica contra Schopenhauer y Wagner, sino como parte del mismo proceso de emancipación, de su devenir filósofo.

\section{LA AUTO-PERCEPCIÓN DEL DESARROLLO CONTINUO.}

En esta sección nos ocuparemos de mostrar la idea de continuidad que Nietzsche expresa al hablar de su trayectoria, de su vida y al presentarse al mundo, tras la fase wagneriana. Como ha argumentado Sánchez Meca en El itinerario intelectual de Nietzsche, en los años de formación y profesión filológica ya se aprecia una orientación en la crítica que Nietzsche ejerció en 1869 a través de la lección sobre Homero y la filología clásica, que «continúa luego en todos sus estudios y publicaciones posteriores sobre el mundo griego»: «una revisión de la imagen filológica oficial de la Antigüedad que distingue en ella el signo del síntoma, la voluntad y la interpretación» ${ }^{57}$. Dicho de otro modo, hay un germen o anticipo de la posterior genealogía, por ejemplo cuando se conceptualizan las interpretaciones como síntomas, si bien utiliza como coordenada no el cuerpo, sino la metafísica de la voluntad schopenhaueriana, que como sabemos pronto será abandonada. Del mismo modo, la crítica que Nietzsche despliega en las obras consideradas período intermedio, ilustrado o «positivista» son el desarrollo coherente y la maduración de la crítica ya

52 Ibid., p. 239.

53 Ibid., p. 238.

54 A $\$ 429$, OC III 651-652; A §482, OC III 665-666; GC §3, OC III 741-742; GC §107, OC III 793; GC §123, OC III 799-800. Mazzino Montinari, «Nietzsches Philosophie als 'Leidenschaft der Erkenntnis'», en Nietzsche lesen, cit., pp. 64-78; Marco Brusotti, Die Leidenschaft der Erkenntnis. Philosophie und ästhetische Lebensgestaltung bei Nietzsche von Morgenröthe bis Also sprach Zarathustra, Berlin-New York: de Gruyter, 1994.

55 FP III 271, 12[1] (33).

56 HDH §164, OC III 150.

57 Diego Sánchez Meca, op. cit., p. 155. 
ensayada en sus escritos de juventud, por ejemplo en La filosofía en la época trágica de los griegos ${ }^{58}$. En este escrito, hay un ansia de «recrear aquellas naturalezas mediante una comparación» 59 , anticipando los presupuestos de su investigación histórica y la perspectiva de la experimentación, que emergerán con fuerza en la filosofía del espíritu libre. En la primera introducción de este texto se destaca que los elementos de cada sistema filosófico «forman parte de una personalidad» y que su estudio contribuye a «la tarea» de desvelar «aquello que debemos siempre amar y venerar (...): el gran hombre» ${ }^{60}$. También se advierte una reflexión de orden ya fisiológico, como se muestra en la reflexión sobre la interdependencia entre la filosofía y la salud de un pueblo $^{61}$. El hilo conductor de toda la filosofía de Nietzsche es su reflexión sobre la humanidad, su historia, su presente, sus potencialidades, su futuro, así como el influjo que tienen las distintas áreas de la cultura — desde el arte hasta la política-en la formación como en la transformación de los seres humanos. Ya en su juventud se había percatado de «la más profunda necesidad de otra imagen del hombre», pero eso no quitaba el «malentendido de mi juventud: todavía no me había desembarazado completamente de la metafísica» ${ }^{62}$.

Este progresivo desembarazo de la metafísica, y la presencia cada vez más importante de la fisiología, se aprecia en una importante carta de 1881. En ella, transmite a Overbeck el gran entusiasmo que le despierta su libro Aurora, calificándolo como «el principio de mis comienzos ¡cuántas cosas tengo aún ante mí! ¡y cuántas sobre mis espaldas!», además de una explícita voluntad de concentrar su tiempo y atención en los «estudios de fisiología y de medicina (¡sé tan poco sobre ello! - ¡y son tantas las cosas que necesito saber de verdad!)» ${ }^{63}$. A diferencia de Humano, demasiado humano, Aurora no se ocupa de la historia de las ideas, sino más bien de la de los sentimientos morales, reflexión que en gran medida está estimulada por John Stuart Mill, Auguste Comte, Herbert Spencer, Émile Littré y Julius Baumann. Aurora representa además una investigación crítica con las propuestas de fundamentación tradicionales, basadas en el deber (Kant), la utilidad (Spencer) o la compasión (Schopenhauer) ${ }^{64}$. Así lo expresa de nuevo en el prólogo de 1886, donde reitera esta idea: en Aurora «bajé a las profundidades, horadé hasta el fondo, como si se tratara del suelo más firme, comencé a indagar y a cavar en la confianza

58 Ibid.

59 FTG «Introducción 1», OC I p. 571.

60 Ibid.

61 FTG $\S 1$, OC I 573.

62 FP III 630, 27[78].

63 Carta $n^{\circ} 139$ de 1881 , CO IV p. 147. En esa misma carta le encarga a Overbeck la compra de cinco libros para desarrollar su doctrina del eterno retorno en relación con las hipótesis cíclicas sobre el tiempo.

64 Mazzino Montinari, «'Aurora’ nell’opera di Nietzsche», cit., p. 628. 
antigua sobre la que los filósofos, como si se tratara del suelo más firme, habíamos estado construyendo los últimos dos mil años (...) la confianza que tenemos en la moral» ${ }^{65}$. En Aurora se instaura ciertamente la incorporación de un nuevo punto de vista sobre el origen y la naturaleza de la moral, que encauza la reflexión hacia el cuestionamiento del valor de los valores, hacia lo que posteriormente elaborará en De la genealogía de la moral ${ }^{66}$.

En un fragmento preparatorio del prólogo de L'Ombra di Venezia, obra que después se convertiría en Aurora, Nietzsche escribe:

Cuando siendo más joven hice la prueba de releer escritos míos que había olvidado, me asustó un rasgo común de todos ellos: hablan el lenguaje del fanatismo. En casi todos los lugares en que se habla de quienes piensan de manera distinta, llaman la atención ese modo sangriento de renegar y ese entusiasmo en la maldad que son los signos del fanatismo, - signos odiosos, a causa de los cuales no habría soportado leer hasta el final esos escritos, y hubiese conocido un poco menos al autor. El fanatismo arruina el carácter, el gusto y en definitiva la salud; y quien quiera reestablecer los tres de manera radical, tiene que estar dispuesto a pasar por una cura larga y aburrida.

Tras haber dicho tanto de mí mismo, y no precisamente lo más edificante como la costumbre del prólogo no aconseja pero sí permite- puedo esperar al menos haber logrado que mis pensamientos más recientes, que en el presente libro doy a conocer, se lean no sin precaución ${ }^{67}$.

Este comentario entra en consonancia con las preocupaciones e intereses filosóficos que protagonizan tanto Humano, demasiado humano como Aurora: la indagación de las construcciones gnoseológicas, sociales y morales y el desenmascaramiento de la apariencia absoluta o suprahistórica de lo que en realidad es siempre relativo al ser humano, mortal, frágil y efímero. Nietzsche reconoce esta situación de crisis de los valores antaño tenidos por sagrados e inmutables como un resultado positivo del despliegue de la razón moderna, porque abre espacio a la crítica del dogmatismo, y a la imposibilidad de reducir todas las perspectivas a una única, tomada como válida y verdadera, y de la estructura relacional entre discípulo y maestro cuando éste se convierte en un ídolo. La crisis de estos tres elementos es un efecto nihilista de la muerte de Dios que Nietzsche celebrará en el libro inmediatamente posterior, La gaya ciencia. La incitación final a ser leído con precaución, a modo de advertencia, no hace sino resaltar el carácter situado y provisorio de todo pensamiento,

65 A «Prólogo» §2, OC III 484.

66 Maria Cristina Fornari, «'Ein Kampf, der nicht der Kampf der Motive ist'. Aurora como reale punto di svolta nella riflessione nietzscheana», en Céline Denat y Patrick Wotling (eds.), Aurore, tournant dans l'oeuvre de Nietzsche?, Reims: Épure, 2015, pp. 54-63.

67 FP II 515, 3[1]. 
susceptible de ser mejorado en el futuro o modificado a la luz de nuevo argumentos o de nuevas circunstancias.

Mención especial en este análisis merecen los prólogos de 1886 de las reediciones de sus obras anteriores que Nietzsche desea republicar. Ese año escribe a su editor Fritzsch que tras el paso de los años y con la debida distancia se siente por fin preparado para redactar prólogos para los dos tomos unificados de Humano, demasiado humano, Aurora y La gaya ciencia. Mediante los prólogos, desde el El nacimiento de la tragedia hasta el prólogo de De la genealogía de la moral publicado ya en su primera edición, quiso trazar «una especie de "historia evolutiva"» ${ }^{68}$. Aseveraciones como esta ilustran con claridad que Nietzsche no concibió su progreso en términos de ruptura, sino como desarrollo, crecimiento, avance y ejercicio: un despliegue que no habría sido posible sin los estadios anteriores. Nietzsche justificó del siguiente modo la necesidad de incorporar dichos prólogos pasados los años:

Ahora, cuando yo mismo puedo decir de la mejor manera y del modo más preciso qué es lo que constituye lo propio e incomparable de esas obras y en qué medida inauguran para Alemania una nueva literatura (el preludio de una cultura y autoeducación moralista que hasta ahora ha faltado a los alemanes), me decidiría con gusto a escribir esos prólogos posteriores, que miren hacia atrás. Mis escritos presentan un desarrollo continuo, que no será sólo mi vivencia y mi destino personal: - sólo soy el primero, una generación emergente comprenderá por sí misma lo que yo he vivido y tendrá un fino paladar para mis libros. Los prólogos podrían poner de manifiesto lo que hay de necesario en el curso de ese desarrollo: lo que de paso tendría la utilidad de que quien ha hincado el diente en uno de mis escritos, tendrá que vérselas con todos ${ }^{69}$.

Partiendo de la constancia de la dificultad de sus obras ${ }^{70}$, Nietzsche quiso delinear con sumo cuidado los sentidos y aclarar el mensaje de cada uno de esos libros, por ejemplo, la unificación de Humano, demasiado humano en dos tomos tiene como parte de un mismo proceso de cura espiritual, «enseñanza de la salud» que quiere compartir con las nuevas generaciones, de «autotratamiento antirromántico», contra la «enfermedad temporal» ${ }^{71}$ de la fase wagneriana. Hay una interpelación a sus lectores y a los buenos europeos, a quienes quiere enseñar «el arte serpentino de mudar la piel», a deshacerse

68 Carta $^{\circ} 908$ de 1885 , CO V p. 363.

69 Carta n $^{\circ} 730$ de 1886 , CO IV p. 199.

70 GC $\$ 371$ y GC $\$ 381$ (quinto libro de 1887), OC III 886 y 892.

71 HDH II «Prólogo» §2, OC III 276. 
del romanticismo ${ }^{72}$, y también como «maestro de la lectura lenta», pues «no por nada ha sido uno filólogo» ${ }^{73}$.

La atención que dedica en 1886 a las obras del considerado período intermedio revela esta misma noción de continuidad, no de rechazo, sino de desarrollo, siempre desde la conciencia de su intempestividad y con vistas a favorecer la comprensión de sus lectores, que han de aprender a leerle bien: «tengo que dar una serie de premisas educativas hasta que por fin haya criado mi propio lector (bis ich mir endlich meine eignen Leser gezüchtet habe), es decir, lectores que puedan ver mis problemas sin ser destruidos por ellos» ${ }^{74}$. Esta atención a los lectores se explica en parte por su aseveración de que el sentido histórico le hacía sentir en sus hombros el peso de «la "humanidad" futura», como un eslabón o como el germen de una planta maravillosa ${ }^{75}$.

A partir de De la genealogía de la moral, el interés de Nietzsche se desplaza de la pregunta por el origen de la moral y de los valores, a la interrogación y examen del valor mismo de dichos valores y su sentido. La investigación consiste en determinar en cada caso, las condiciones en que un juicio de valor ha surgido, ha sido inventado, pero también, y sobre todo, en qué medida el valor de dichos juicios ha influido en el desarrollo del propio ser humano hasta el presente. Este es el criterio expresado el prólogo del libro ${ }^{76}$. La genealogía es la indagación por la proveniencia de los valores junto con su evaluación en relación con su impacto beneficioso o dañino en el florecimiento humano, «su plenitud, su fuerza, su voluntad de vida, su confianza y su futuro» ${ }^{77}$, una reflexión sobre el valor vital. Esta crítica es histórica y se construye sobre la historia natural de la moral y de dichos valores, elaborada en las obras anteriores, antesala necesaria, que, por otro lado, carecían de este componente radicalmente crítico. Por tanto, la genealogía es una continuación de la reflexión histórica cuyo germen nació en la segunda Consideración intempestiva y que fue desarrollada con explicitud a partir de Humano, demasiado humano y Aurora, que en palabras del propio Nietzsche representaba el comienzo de su «campaña contra la moral» ${ }^{78}$. Más allá del bien y del mal, obra inmediatamente anterior a De la genealogía de la

72 Ibid. Cf. HDH II «Prólogo» §6, OC III 279.

73 A «Prólogo» 5, OC III 488-489.

74 Carta $^{\circ} 761$ de 1886, CO V 228

75 GC $\$ 377$, OC III 853-854.

76 GM «Prólogo» §3, OC IV 454-455.

77 Ibid., 455

78 EH «Aurora» §1, OC IV 830. Montinari se mostró escéptico con esta caracterización, que aunque refleja la «intención central de la obra, no es del todo exacta con respecto al desarrollo el pensamiento de Nietzsche», pues esta tarea comenzó en parte en Humano, demasiado humano (Mazzino Montinari, «'Aurora' nell'opera di Nietzsche», en Opere di Friedrich Nietzsche, a cura di G. Colli e M. Montinari, Milano: Adelphi, 1964, vol. 5/1, pp-627-630, p. 627). 
moral, dedicaba su quinto capítulo a «Para la historia natural de la moral ${ }^{79}$, planteada no como ciencia ni desde la perspectiva de su fundamentación, sino como problema. Contra aquellos filósofos de la moral para quienes la «tarea de descripción» de diferentes pueblos y tiempos pasados pudiera parecer vulgar, plantea que la «colección de materiales» que proporciona la historia es condición de posibilidad la «preparación de una tipología de la moral» ${ }^{80}$, es decir, una clasificación, una jerarquización para su posterior selección.

Todo el prólogo de De la genealogía de la moral ${ }^{81}$ representa una importante reflexión de Nietzsche sobre su trayectoria, sus antiguos maestros ${ }^{82}$ y las influencias de sus amistades, por ejemplo Paul Rée ${ }^{83}$, e incluso sobre las dificultades que acarrea la escritura en aforismos para la interpretación de sus escritos $^{84}$. De todo el prólogo, comentaré el segundo parágrafo, en el que medita sobre la madurez alcanzada desde Humano, demasiado humano, como signo de su fortaleza ${ }^{85}$. En primer lugar, afirma que los pensamientos expresados en Humano, demasiado humano surgieron incluso con anterioridad al viaje a Sorrento. Ello refuerza la hipótesis del contraste entre lo que Nietzsche publicó durante la fase wagneriana y lo que realmente ocupaba su mente, es decir, cuáles eran sus intereses y pensamientos filosóficos. En segundo lugar, no reniega de Humano, demasiado humano: los pensamientos «en esencia eran ya los míos que retomo en los tratados presentes» de De la genealogía de la moral, únicamente desea que, con la ventaja que proporciona el paso del tiempo y la madurez, ser capaz de fortalecer tanto el contenido como la forma. En tercer lugar, siguiendo la imagen del árbol, expresa que todos sus frutos son «similares y relacionados entre sí, testigos de una única voluntad, de una salud única» ${ }^{86}$, nada más alejado de la concepción tripartita llevada a su hipérbole.

\section{ECCE HOMO, LA ESTRATEGIA DE LA AUTOPRESENTACIÓN.}

Ecce Homo es una obra extremadamente particular que despierta sentimientos encontrados dentro de los especialistas dado el tono burlesco del escrito y las autoalabanzas que parecen premonitoras de la demencia ${ }^{87}$.

79 MBM $\S 186$ ss., OC IV 353-368.

80 Ibid., 354.

81 GM «Prólogo» §4, OC IV 453-459.

82 GM «Prólogo» §4, OC IV 457.

83 Ibid., 455-456.

84 GM «Prólogo» §8, OC IV p. 459.

85 GM «Prólogo» §2, OC IV 454.

86 Ibid.

87 Cf. el «Prefacio» de Manuel Barrios a Ecce Homo, en OC IV 775-780. Barrios puntualiza con atino que esas alabanzas son en realidad una recopilación de los elogios que Nietzsche recibió por parte de Taine, Strindberg y Widmann. Cf. nota 10, p. 779. 
Quizás Nietzsche era consciente del deterioro de su salud y sentiría con más ánimo la urgencia de presentarse. Era perfectamente consciente de que «el libro es rico en bromas y maldades, porque yo me presento con toda violencia como el tipo antitético de la especie de ser humano que ha sido venerada hasta ahora: - el libro es lo más "profano" posible.... ${ }^{88}$.

Dentro de las numerosas aristas del libro, me propongo en el espacio que queda presentar algunas líneas de fuga sobre Ecce Homo como estrategia de autopresentación, más concretamente, qué nos dice esta obra de su visión sobre su trayectoria intelectual, si acaso se aprecia una periodización de sus obras en este escrito. Ecce Homo retoma el mismo cometido de los prefacios de 1886 y 1887 , con los que Nietzsche quiere perfilar su imagen como filósofo y solventar algunos malentendidos, como se muestra en el comentario aclaratorio sobre el superhombre frente a la interpretación en clave darwinista ${ }^{89}$, pero siempre dejando espacio al juego de máscaras y también a la reflexión hermenéutica, para acabar, no sin malicia, con un «-iSe me ha comprendido? - Dioniso contra el crucificado...» ${ }^{90}$. Esta obra representa además su voluntad de abandonar su vida de filósofo errante, levantar acta de ciertos elementos de su biografía y presentarse al mundo con claridad como una suerte de prólogo antes de la Transvaloración de los valores ${ }^{91}$. En consonancia con la fuerte personalización de su filosofía en los últimos años, hay una clara intención de encarnar el corpus de sus obras ${ }^{92}$ y de plantear con gran explicitud la tarea. El libro comienza expresando con claridad el objetivo de la obra — con sospechas casi premonitorias de la posterior manipulación nacionalsocialistas de su obra-, expresa su necesidad de insistir en «quién soy yo», pese a no haber cesado de «dar testimonio de mí», con el claro deseo de no ser confundido ${ }^{93}$. El segundo párrafo del prólogo insiste en cómo su «oficio» representa una antítesis frente a la pretensión de «"mejorar" a la humanidad», que como sabemos por obras anteriores, es el término con el que Nietzsche designa lo que la religión y la moral ascética han pretendido hacer con el ser humano, y cómo «la humanidad misma ha resultado engañada y falseada» por la mentira de los ideales ascéticos «hasta en sus más básicos instintos», a través de la inoculación de los «valores inversos a los únicos con los que estarían garantizados la prosperidad, el futuro, el alto derecho al

88 Carta $^{\circ} 1157$ de 1888 , CO VI 303.

89 EH «Por qué escribo tan buenos libros» §1, OC IV 810.

90 EH «Por qué soy un destino» §9, OC IV 859. cf. Carta n ${ }^{\circ} 1137$ de 1888, CO VI 281.

91 Maria Cristina Fornari, op. cit., p. 283 y p. 293.

92 Giuliano Campioni, «Introduzione» a Nietzsche, Friedrich, Lettere da Torino, Milano: Adelphi, 2008, p. 20.

93 EH «Prólogo» §1, OC IV 781. 
futuro» ${ }^{94}$. No es una desaparición del valor, sino su inversión.

La «advertencia preliminar» es también un pasaje significativo en el seno de nuestra argumentación a favor de la continuidad. Nietzsche expresa: «acaba de posarse un rayo de sol sobre mi vida: he mirado hacia atrás, he mirado hacia adelante, y nunca había visto de una sola vez tantas y tan buenas cosas. (...) ¿Cómo no debería estar agradecido a mi vida entera? Y así me cuento mi vida a mí mismo» ${ }^{95}$. En sus palabras no se aprecia un rechazo, sino la aceptación, quizás tras la incorporación de la doctrina del eterno retorno y el amor fati. No hay aversión y desprecio, sino la aprobación de aquello que ya ha pasado. Cada instante forma parte del conjunto del proceso, su existencia y vida misma ha sido también posible gracias a la cadena de todo lo que ha ocurrido, en el juego de la realidad. También la fase wagneriana fue condición necesaria de su desarrollo. Querer una cosa significa quererla en totalidad, incluso sus aspectos negativos. Incorporar la doctrina del eterno retorno significa querer revivir la vida incluso en sus elementos más crueles ${ }^{96}$. Así, el querer, como conversión afectiva, se libera. Zaratustra, como tipo psicológico, es redentor: es «alguien que dice sí hasta la justificación, hasta la redención de todo lo pasado» ${ }^{97}$.

Ecce Homo expresa, pues, el desarrollo personal del cual sólo se es consciente a través de la retrospección, pero sobre todo, es una recapitulación con la función de preparar para sus futuras obras. Desde el privilegio que proporciona el paso del tiempo, la narración del desarrollo personal tiene como misión destacar en sus libros una importante coherencia interna, una orientación, que expresa bajo la imagen del «destino» ${ }^{98}$, como su misión, más allá de la habitual mención de su «tarea» (Aufgabe), ahora reforzada con un destacable cariz de necesidad o incluso de «fatalidad» en su vida ${ }^{99}$, del carácter irremediable con el que gesta sus escritos, Ecce Homo incluido, que son «síntomas y, a la vez resultado, de lo que él mismo es» ${ }^{100}$. Así, Richard Wagner en Bayreuth sería «una visión de mi futuro]» y Schopenhauer como educador «mi historia más íntima, mi devenir. ¡Sobre todo, mi solemne promesa!»101.

94 EH «Prólogo» §2, OC IV 782.

95 EH, OC IV 784.

96 Za IV «La canción del noctámbulo» §10, OC IV 276.

97 EH «Así habló Zaratustra» §8, OC IV 841.

98 Carta $\mathrm{n}^{\circ} 1151$ de 1888 , CO VI 297 y carta n ${ }^{\circ} 1137$ de 1888 , CO VI 281.

99 EH «Por qué soy tan sabio» §1, OC IV 785; Carta nº 617 de 1885, CO V 87: «Quizás llegue alguien que descubra que a partir de Humano demasiado humano no he hecho otra cosa más que cumplir mis promesas».

100 Maria Cristina Fornari, «Talis hominibus fuit oratis qualis vita. Nietzsche y la narración de sí», loc. cit., p. 37.

101 EH «Las Intempestivas» §3, OC IV 824. 
El capítulo dedicado a Humano, demasiado humano, como ya se ha entrevisto en la sección anterior, narra con detalle la ruptura con Wagner, con su vida de filólogo, y en general, el cambio de orientación hacia la filosofía informada por la fisiología, la medicina y las ciencias naturales. También señala la ignorancia propia de la «juventud», la indolencia y el sentido de deber como los motivos del desinterés con el que claudicó ante otros, los siguió o se confundió con ellos ${ }^{102}$, en clara referencia a las Intempestivas dedicadas a Schopenhauer y Wagner en las que ahora se reconoce a sí mismo y no a ellos. Es en 1876 cuando Nietzsche toma las riendas de su vida, publica el «monumento al riguroso autocultivo (Selbstzucht)» ${ }^{103}$, y asume su tarea. Dicha tarea es expresada en el capítulo dedicado a Aurora con una explicitud mucho mayor de aquella con la que se expresa en el propio libro:

Mi tarea de preparar a la humanidad para un instante de suprema autoconciencia, para un gran mediodía en el que volver la vista atrás y mirar hacia delante, en el que escapar del dominio del azar y de los sacerdotes y plantear por primera vez, como un todo, la cuestión del por qué, del para qué, — esa tarea se sigue necesariamente de la convicción de que la humanidad no marcha por sí misma por el camino correcto, que no es regida en absoluto por una divinidad, sino que más bien, por el contrario, bajo sus más sagrados conceptos de valor se ha dejado gobernar seductoramente por un instinto de negación, de corrupción, por un instinto de décadence. La cuestión del origen de los valores morales es por eso, para mí, una cuestión de primer orden, porque condiciona el futuro de la humanidad ${ }^{104}$.

Hay en este pasaje varios elementos destacables: la crítica a la idea de selección natural del darwinismo, del azar en la historia, del sentido de la humanidad, etc. Aurora era una obra filosófica sobre el desarrollo histórico de la moralidad — las costumbres-, el pensamiento, los sentimientos morales, los prejuicios filosóficos sobre el sujeto y la moral altruista, además de algunas consideraciones sociales, económicas y políticas. Ya hay tímidas referencias a la idea de selección o cultivo, sobre todo expresadas a través de la metáfora del jardín y el jardinero ${ }^{105}$, pero no de esa manera. En esta cita Nietzsche pone de relieve las aportaciones de Aurora, el análisis de los valores morales, por ser un paso necesario para la posterior transformación de la humanidad y transvaloración de los valores. Por ejemplo, ya hay en Aurora importantes

102 EH «Humano, demasiado humano» §4, OC IV 827.

103 EH «Humano, demasiado humano» §5, OC IV 828.

104 EH «Aurora» §2, OC IV 831.

105 Cf. la imagen del pensador como jardinero en Aurora: A §382, OC III 641, y del manejo de las pulsiones como «semillas» en A §560, OC III 691. 
análisis sobre la proveniencia (Herkunft) de los juicios y los sentimientos en que éstos se basan, nos dice que «los sentimientos no son lo último, lo originario; detrás de los sentimientos hay juicios y valoraciones que se nos han transmitido por herencia en forma de sentimientos (inclinaciones, aversiones)» ${ }^{106}$. No es «tuyo» el juicio que se funda en esos sentimientos ${ }^{107}$, pertenece a toda la línea evolutiva, el linaje, que ha recorrido hasta llegar a ti, elementos necesarios para la «suprema autoconciencia». La crítica a la Modernidad que se va desarrollando desde los escritos de juventud hasta la filosofía histórica de Humano, demasiado humano, Aurora y La gaya ciencia, obtiene un momento marcadamente afirmativo a partir de $A s i$ habló Zaratustra, donde los discursos sobre la transformación y superación del ser humano obtienen gran presencia. Pero el momento de la crítica fue necesario para la posterior propuesta de transformación, Así, para preparar a la humanidad, como nos dice en Ecce Homo, hay que transformar las cualidades y preferencias hereditarias analizadas en los escritos aforísticos como Aurora.

Una de las características más notables de Ecce Homo es que Nietzsche escribe sobre sus obras de juventud o intermedias empleando el léxico propio de la segunda mitad del decenio de 1880 - conceptos que no existían con anterioridad o cuya presencia era discreta-. Por ejemplo, analiza $E l$ nacimiento de la tragedia desde la óptica del nihilismo y la décadence, criticando aquello que ahora juzga de erróneo en ella, pero señalando, al mismo tiempo, que recuerda «la inmensa esperanza» que yacía bajo este libro fantaseando un «nuevo partido de la vida que tome en sus manos la mayor de todas las tareas, la cría superior de la humanidad» ${ }^{108}$. Es decir, el proyecto de reforma de la cultura antaño formulado como el sueño romántico wagneriano es ahora redefinido en los términos psicofisiológicos propios de la madurez, que en los años de juventud permanecían latentes y ocultos en los cuadernos, junto a otras metáforas más tímidas de la selección. Esta manera de cerrar el círculo, o dicho de otro modo, de conectar el origen con el presente, resalta sin duda la continuidad en la tarea y el sentido de su proyecto filosófico. También señala la tercera y la cuarta Intempestiva como figuras de «la más dura cría de uno mismo» y reprueba a los filisteos de la cultura y librepensadores el querer «conforme a su imagen, "mejorar" a la humanidad» ${ }^{109}$, alusión probable al capítulo de El crepúsculo de los ídolos, de ese mismo año. También en estos capítulos resalta la necesidad de cada uno de los pasos de su itinerario, o más concretamente, de sus funciones o profesiones: «haber sido muchas cosas y

106 A $\S 35$, OC III 508.

107 Ibid.

$108 \mathrm{EH}$ «El nacimiento de la tragedia» §1, 2, 3, 4, OC IV p. 817-820.

109 EH «Las Intempestivas» §1, 2, OC IV p. 821-823. 
en muchos sitios, para poder llegar a ser una única cosa, _ (...) Durante un tiempo tuve que ser también un erudito. $\longrightarrow\rangle^{110}$.

Más allá de Ecce Homo, en los últimos años se aprecia un reencuentro o convergencia de numerosos conceptos nietzscheanos dentro de un conjunto coherente. Por ejemplo, algunas nociones como lo dionisíaco son reinterpretadas bajo la égida del proyecto de cría. Desde su juventud, Nietzsche instaura un modo de pensar dionisíaco, que consiste en poner en evidencia la ilegitimidad de las oposiciones dicotómicas, en realidad conectadas por una solidaridad profunda. En los últimos años de su vida retoma la figura de Dionisos ${ }^{111}$ para designar «una consideración que - en el crear, transformar, al hombre tanto como las cosas - reconoce el sumo deleite de la existencia (...) y ve en la moral únicamente un medio para dar a la voluntad dominante una fuerza y flexibilidad conforme a los cuales pueda ser formada la humanidad» ${ }^{112}$ basado en la consideración de las morales y las religiones como medios para «hacer del hombre lo que uno quiera», en un amplio espacio de tiempo. También en 1888 la ruptura con Wagner es revisitada en Nietzsche contra Wagner, y reconsidera y matiza numerosas tesis, como el tránsito del filósofo eremita al filósofo legislador, de tal manera que el apoliticismo abre paso a la Gran Política $^{113}$. Asimismo hay una reflexión estética en la madurez: la fisiología del arte, de importante cariz político. Estos son sólo algunos ejemplos de la labor de reconstrucción narrativa y unificación retrospectiva apreciable en los últimos años, fruto de una voluntad de resituar debidamente el conjunto de sus pensamientos, hacerse comprensible y presentarse a sus soñados lectores: los buenos europeos ${ }^{114} \mathrm{y}$ los filósofos del futuro.

\section{CONCLUSIONES}

Con este artículo, se han querido presentar diversos elementos que permiten flexibilizar o relativizar la tradicional periodización de la filosofía de Nietzsche, a favor de una perspectiva que privilegie la lente de la continuidad, avalada además por la autoconciencia de su desarrollo, en el que sólo se reconoce una fase, e incluso esa misma fase es rememorada como secuencia de una trayectoria, necesaria en el proceso de devenir el que es. La significativa retrospección y la constante reflexión sobre su autodesarrollo no indican ruptura o rechazo, sino reconocimiento. Como comentábamos al comienzo, sin duda la periodización cumple efectos pragmáticos: nos permite

110 EH «Las Intempestivas» §3, OC IV 824.

111 FP III 752-753, 34[176].

112 FP III 753, 34[176].

113 Carlo Gentili, «Nietzsche, ¿político o apolítico?»: Estudios Nietzsche 12 (2012), 105-116.

114 Pietro Gori y Paolo Stellino, «'Los dueños de la tierra, los legisladores del futuro’: Los buenos europeos de Nietzsche y la renovación cultural de Europa»: Estudios Nietzsche 15 (2015), 45-61. 
comunicarnos, organizar y reflexionar sobre los distintos momentos o escritos de Nietzsche, pero es un artificio de los estudios nietzscheanos. Por ello, no debería convertirse en dogma una perspectiva de discontinuidad que el propio Nietzsche no practicaba a la hora de narrarse a sí mismo su vida y sus obras. Para no perder de vista el itinerario que representa el pensamiento de Nietzsche, y al mismo tiempo ser capaces de aislar un tema concreto para analizarlo en profundidad en el seno de una diacronía dinámica, es necesario seguir su génesis escritural en los manuscritos, su posterior filiación y transformación conceptual, su apoyo en las fuentes y el análisis de sus lecturas, para seguir la historia de un complejo temático y su manera de articularse con otros temas ${ }^{115}$. La crítica genética, unida a la hermenéutica crítica ${ }^{116}$, permiten estudiar la historia de las metamorfosis temáticas y su manera de combinarse con los diversos problemas de la filosofía nietzscheana. A lo largo de su producción hay numerosos elementos de relación, que se aprecian sobre todo en la orientación de su filosofía, su tarea: la crítica de la cultura, la transformación del ser humano y la superación del nihilismo.

115 Paolo D’Iorio, «Système, phases diachroniques, strates synchoniques, chemins thématiques», loc. cit., pp. 35-36.

116 Jesús Conill, El poder de la mentira. Nietzsche y la política de la transvaloración, Madrid: Tecnos, 2007, $3^{\text {a }}$ ed., segunda parte: «Nietzsche y la hermenéutica contemporánea». Jesús Conill, Ética hermenéutica. Crítica desde la facticidad, Madrid, Tecnos, 2010. 
